Canadian Art Review

\title{
Graphisme et praxis chez Ernest Cormier, " architecte et ingénieur-constructeur " : le " pavillon principal " de l’Université de Montréal
}

\section{Isabelle Gournay}

Volume 16, numéro 2, 1989

Études sur l'architecture et son environnement

Studies on Architecture and its Environment

URI : https://id.erudit.org/iderudit/1073147ar

DOI : https://doi.org/10.7202/1073147ar

Aller au sommaire du numéro

\section{Éditeur(s)}

UAAC-AAUC (University Art Association of Canada | Association d'art des universités du Canada)

\section{ISSN}

0315-9906 (imprimé)

1918-4778 (numérique)

Découvrir la revue

\section{Citer cet article}

Gournay, I. (1989). Graphisme et praxis chez Ernest Cormier, « architecte et ingénieur-constructeur » : le " pavillon principal » de l'Université de Montréal. RACAR : Revue d'art canadienne / Canadian Art Review, 16(2), 161-164.

https://doi.org/10.7202/1073147ar
Résumé de l'article

This article examines drawings for the "main pavilion" of the Universite de Montréal built on Mount Royal between 1928 and 1943. A collection of these drawings will be exhibited at the CCA in the spring of 1990. Cormier's cosmopolitan background as both an architect and an engineer is mentioned briefly, as is the evolution of the project from a traditional academic ensemble of separate pavilions to the present "megastructure." This dramatic change is demonstrated by the modernization of drawing techniques taught at the Ecole des Beaux-Arts in Paris and the replacement of large renderings in watercolours by photographs of the preparatory model as a promotional medium. All of Cormier's drawings reflect his French-inspired approach to design and stress the logical expression of both structure and ornamentation. The 1,300 working drawings for the final project form an exceptionally homogeneous ensemble that reveals the architect's tight control over his small team of highly qualified draughtsmen and his personal involvement at every stage of the design process.
Tous droits réservés @ UAAC-AAUC (University Art Association of Canada | Association d'art des universités du Canada), 1989
Ce document est protégé par la loi sur le droit d'auteur. L'utilisation des services d'Érudit (y compris la reproduction) est assujettie à sa politique d'utilisation que vous pouvez consulter en ligne.

https://apropos.erudit.org/fr/usagers/politique-dutilisation/ 


\title{
Graphisme et praxis chez Ernest Cormier, "architecte et ingénieur-constructeur»: le «pavillon principal» de l'Université de Montréal
}

\author{
- \\ ISABELLE GOURNAY* \\ Conservateur invitée \\ Centre Canadien d'Architecture/Canadian Centre for Architecture
}

\section{A BSTRACT}

This article examines drawings for the "main pavilion" of the Université de Montréal built on Mount Royal between 1928 and 1943. A collection of these drawings will be exhibited at the CCA in the spring of 1990. Cormier's cosmopolitan background as both an architect and an engineer is mentioned briefly, as is the evolution of the project from a traditional academic ensemble of separate pavilions to the present "megastructure." This dramatic change is demonstrated by the modernization of drawing techniques taught at the Ecole des BeauxArts in Paris and the replacement of large renderings in watercolours by photographs of the preparatory model as a promotional medium. All of Cormier's drawings reflect his French-inspired approach to design and stress the logical expression of both structure and ornamentation. The 1,300 working drawings for the final project form an exceptionally homogeneous ensemble that reveals the architect's tight control over his small team of highly qualified draughtsmen and his personal involvement at every stage of the design process.

\section{UN MAITRE D'OEUVRE ET UN CHANTIER EXCEPTIONNELS}

De 1902 à 1906, Ernest Cormier étudie à l'Ecole Polytechnique de Montréal, sa ville natale, puis il prépare par correspondance le concours d'entrée à l'Ecole des Beaux-Arts de Paris. En 1908, il part pour la France; quelques mois plus tard, il est reçu à ce concours et intègre l'atelier de Jean-Louis Pascal. Son habileté graphique lui vaut des médailles à diverses épreuves de dessin et une mention honorable au Salon des Artistes Français de 1914, où il expose des aquarelles d'Italie ${ }^{1}$. Au delà des techniques de rendu, cette formation académique largement inspirée par les écrits de Julien Guadet

1 Dès 1904, Ernest Cormier s'initie au modelage en fréquentant les cours du soir au Monument National; il en poursuit l'apprentissage à Paris auprès du sculpteur Allard. Ses aquarelles sont très proches de celles de son professeur Pierre Vignal (1855-1925), dont les archives du cca conservent les reproductions dans l'hebdomadaire populaire L'Illustration et, de 1925 à 1929, chez J. H. Jansen à Cleveland.

* Je remercie MM. Desaulniers et Fortier du Centre Canadien d'Architecture pour leur précieux concours. 
a surtout le mérite d'enseigner une approche logique des données d'un programme et une méthode de travail rigoureuse. Ajoutons que la formation européenne d'Ernest Cormier n'a pas eu simplement pour cadre les prestigieuses institutions que sont l'Ecole des Beaux-Arts et la British School à Rome, où il réside en 1915 et 1916. En janvier 1917, confronté aux pénibles réalités de l'effort de guerre, Cormier trouve à s'employer au bureau d'étude Considère, Pelnard et Caquot à Paris. Exploitant les possibilités architectoniques des nouveaux procédés de construction en béton armé et fretté, il réalise plusieurs structures industrielles et hangars d'aviation. Précis mais élégants, les calques et bleus relatifs à ces projets sont la préfiguration des dessins d'exécution examinés dans cet article ${ }^{2}$.

Revenu à Montréal en avril 1918, Ernest Cormier se distingue de ses collègues non seulement par sa formation, mais encore par sa sensibilisation aux nouvelles tendances architecturales. Il se rend fréquemment en Europe et aux Etats-Unis et collectionne des ouvrages et périodiques de référence, anciens et contemporains. Aussi trouvet-on dans certains projets pour l'Université de Montréal des références directes à des bâtiments publiés dans Wendingen ou L'Architecture Vivante. Toutefois, du strict point de vue des modes de représentation graphique, Cormier semble peu perméable aux influences extérieures.

Ernest Cormier est nommé architecte du nouveau campus de l'Université de Montréal en avril 1924. C'est en septembre 1926 qu'il soumet un premier plan d'ensemble qui adopte le parti traditionnel des pavillons séparés (Fig. 185). L'“ ensemble des études "se situe sur un plateau naturel bénéficiant d'une grande visibilité. Au premier plan se trouve la salle des promotions, flanquée de la bibliothèque et des locaux de l'administration (Fig. 186). A l'arrière s'étagent plusieurs ailes de laboratoires (Fig. 187).

En janvier 1927, la Fondation Rockefellerdont on espérait le soutien financierrecommande d'adjoindre à cet ensemble un hôpital universitaire. A la disposition primitive de "l'ensemble des études ", Ernest Cormier cherche donc à greffer vers l'ouest un groupe faculté de médecine/hôpital universitaire apparenté à celui de l'Université de Rochester, qu'il vient de visiter (Figs 188 et 189). Il conçoit une "mégastructure "sur huit niveaux, longue de 280 mètres et concentrant à la verticale les locaux d'administration, les facultés scientifiques et médicales, un

2 Voir par exemple le bleu "Ministère de la Marine. Hangar de $2 \times 220 \times 40$ mètres pour grands ballons dirigeables", plan, coupe, élévation daté du 27 mars 1918. cCA 729A-1,1 729/A-1,1. hôpital universitaire, une tour-bibliothèque et même la chapelle (Figs 190 et 191). Avec la Cathédrale du Savoir de l'Université de Pittsburgh, dont la construction commence en 1926, "l'immeuble de la montagne " manifeste avec éclat une nouvelle direction dans l'architecture universitaire, et symbolise l'ouverture à la modernité de la communauté intellectuelle francophone.

\section{UNE PRATIQUE "HUMANISTE "}

Que savons-nous des méthodes de travail d'Ernest Cormier, dont le papier à en-tête indique clairement la double qualité d' " architecte et ingénieurconstructeur $»$ ? Ne bénéficiant pas de l'infrastructure d'un véritable bureau d'études d'ingéniérie, il ne peut s'imposer en tant que constructeur d'ouvrages d'art. Toutefois, ce sont autant ses compétences techniques que son diplôme de l'Ecole des Beaux-Arts qu'il met en avant pour obtenir des commandes architecturales. Son agence fournit " contrairement à la pratique courante, toutes les quantités d'armatures d'acier, de béton et de coffrage, permettant aux entrepreneurs d'établir un prix de revient des ouvrages serrant de beaucoup plus près la réalité ${ }^{3}$. Ses prestations graphiques sont donc plus complètes que celles des agences québécoises de même envergure, d'où l'importance numérique des dessins d'éxécution pour le pavillon principal de l'Université de Montréal. Numérotés en séries discontinues et a posteriori, on en compte 1037 (le $\mathrm{n}^{0} \mathrm{l}$, indiquant "l'emplacement de la bâtisse ", date du 4 avril 1928; plus du quart d'entre eux sont réalisés en 1930-1931, et 200 dans les années quarante). Leur grande précision n'est certainement pas étrangère à la parfaite mise en oeuvre du pavillon principal, étroitement surveillée par Ernest Cormier et son équipe.

Le registre consignant les dessins d'exécution, dont 47 ont été signés E.C. (Ernest Cormier) (voir Fig. 192), n'indique - toujours sous forme d'initiales - que cinq noms de collaborateurs réguliers. Bien que chargé d'importantes commandes publiques, Cormier demeure tout au long de sa carrière entouré d'une petite et fidèle équipe d'architectes ou d'ingénieurs diplomés ${ }^{4}$. Travailleur acharné, farouchement indépendant, refusant tout compromis avec ses clients, il se réserve le travail de création et les dessins de présentation, et n'est pas de ceux qui oublient l'existence de la planche à dessin. Quand il s'agit de dessins exécutés par un

3 Lettre d'Ernest Cormier à Mgr Piette le 11 avril 1927 (Archives de l'Université de Montréal D 35/120).

4 Les initiales qui reviennent le plus souvent sur les inventaires sont celles de Willford A. Gagnon, le frère du peintre Clarence Gagnon, et d'Edouard Vloeberghs, un ingénieur belge. 
de ses collaborateurs, tels que Willford Arthur Gagnon (Figs 195 et 196), c'est Cormier qui lui donne des directives, sans doute très strictes. Tout au long du projet, Cormier intervient à chaque étape, surveillant de très près le travail de dessinateurs hautement qualifiés.

Ce type de pratique, qu'on peut qualifier d' "humaniste", est plus proche des agences d'architectes français avec qui Cormier fit ses études que des méthodes "taylorisées" des "usines à plans" nord-américaines. La production de l'agence étant étroitement contrôlée, les prestations graphiques pour le pavillon principal forment un ensemble d'une homogénéité exceptionnelle. Leurs formats varient peu, ainsi que leur support (papier calque d'excellente qualité pour les dessins d'exécution). La facture, inspirée du graphisme rigoureux d'Ernest Cormier, est particulièrement uniforme, même à quinze ans d'intervalle.

\section{LA TRADUCTION GRAPHIQUE ET PHOTOGRAPHIQUE DE L'EVOLUTION DU PARTI}

Uniques témoignages du parti adopté pour «l'ensemble des études " en septembre 1926, les dessins de présentation d'Ernest Cormier s'inscrivent en droite ligne dans la tradition des grands rendus à l'aquarelle de l'Ecole des Beaux-Arts. La présence de sangles au dos de l'un d'entre eux indique bien qu'ils étaient destinés à être exposés. La mention "reproduction interdite " est apposée sur les énormes cartouches, agrémentés d'une guirlande stylisée caractéristique du style Art Déco du début des années vingt. Du plan d'ensemble - à l'échelle de 480:1, l'original devait mesurer plus de 3.3 mètres de long - nous ne conservons qu'un photostat (Fig. 185). Faisant référence à des types architecturaux bien établis (et ne rendant pas compte de la forte déclivité du terrain), les perspectives très théâtrales semblent davantage destinées à gagner la confiance des universitaires et du comité de construction qu'à définir un parti. Il est malaisé de trouver un dénominateur commun entre les "façades principales " (Fig. 186) et les " ailes de laboratoires " (Fig. 187) de «l'ensemble des études".

Au contraire, pour le plan d'ensemble définitif, connu également à travers un photostat, Ernest Cormier simplifie et actualise le graphisme (un tel rendu pourrait d'ailleurs être encore pratiqué de nos jours) (Fig. 190). Les indications en caractères romains ont fait place à une typographie des plus contemporaines. Les frondaisons, généreusement figurées au lavis d'aquarelle dans le plan de 1926 , ont été remplacées par de fines hachures et des courbes imbriquées (selon toute probabilité à l'encre ou/et à la mine de plomb), indiquant quelques bouquets d'arbres dans le bas du terrain uniquement-figuration bien plus réaliste! Le tracé des ombres est plus franc; les circulations se lisent mieux. Des aires de stationnement, que rend indispensable l'adjonction d'un hôpital universitaire, font leur apparition, tandis que sont éliminés deux éléments très "Beaux-Arts " de la composition primitive : un petit oratoire sur l'avenue Maplewood - aujourd'hui Edouard-Montpetit et un amphithéâtre de plein air derrière l'ensemble de l'habitation. Cette spectaculaire évolution du parti et du graphisme reflète bien l'esprit "scientifique " qui pousse les universitaires et leur architecte à adopter des dispositions sans précédent pour "l'ensemble des études".

Dessinées au crayon sur papier calque, quelques études de façade à grande échelle pour le corps central et la tour ont été trouvées pliées parmi des documents textuels dans les archives du cca. Tout laisse à penser qu'un grand nombre de ces documents de travail, d'une grande précision mais auxquels l'architecte ne semble avoir accordé aucune valeur artistique, ont disparu. Dans sa version définitive, le pavillon principal de l'Université de Montréal ne fait l'objet d'un dessin de présentation à l'aquarelle qu'a posteriori, lorsqu'Ernest Cormier soumet un morceau de réception à l'Académie Royale du Canada en 1931. En janvier 1928, une maquette en carton, d'aspect relativement grossier mais tout à fait parlante pour le grand public, est montrée dans la salle du Conseil Universitaire. A partir de cette date, ce sont des photographies de la maquette, disparue aujourd'hui (Fig. 191), qui servent à diffuser le projet d'Ernest Cormier. Pris par le studio Hayward, les clichés sont d'excellente qualité (le cca conserve plusieurs tirages de grande dimension, sur fond sépia) et sont les seuls témoins des modifications apportées aux couronnements de la chapelle et de l'aile des amphithéâtres, ainsi qu'aux " solaria " de l'hôpital universitaire. Dans les archives du cca, on note également la présence de nombreuses et fort spectaculaires photographies de chantier, fréquemment reproduites dans la presse populaire québécoise (lors de l'interruption des travaux, ces prises de vues "héroïques" devinrent des instruments de "contre-propagande " destinés à prouver la mégalomanie des universitaires). Pourquoi donc les photographies se sont-elles substituées aux traditionnels dessins de présentation? Aucun document textuel ne permet d'attribuer à coup sûr cette nouvelle orientation à un parti-pris de réalisme et de modernité, ou tout simplement à des impératifs économiques. Mais on a tout lieu de croire que l'Université de Montréal et son architecte eurent vite fait de réaliser le caractère excep- 
tionellement photogénique de "l'immeuble de la montagne " et la nécessité de satisfaire un nouvel appétit d'images de la part d'une société québécoise en pleine mutation.

Ernest Cormier semble également avoir trouvé une alternative réaliste et moderne aux traditionnels rendus à l'aquarelle dans les grands dessins à l'encre sur toile, appartenant à la série non indéxée (et à notre connaissance inédite) intitulée "Façades et coupes" (Figs 193 et 194). Figurant la partie centrale de l'édifice dans sa totalité, leur échelle est trop petite, leur cotes sont trop peu lisibles pour qu'ils servent d'outils de travail aux entreprises. Le rendu, probablement effectué par Ernest Cormier lui-même, est extrêmement soigné, les matériaux de parement (brique et marbre) étant clairement exprimés. Qui plus est, amoureux du travail bien fait et peu avare de son temps et de celui de ses employés, Ernest Cormier fournit des dessins d'éxécution (Figs 195 et 196) d'une qualité plastique exceptionnelle. Tout comme les planches soumises à l'occasion des divers concours de construction à l'Ecole des Beaux-Arts, chaque dessin est une composition, où s'équilibrent plans, coupes et façades à différentes échelles, parfois au détriment d'une lecture aisée sur le chantier.

\section{METHODE ACADEMIQUE. FT LOGIQUE CONSTRUCTIVE}

L'examen des dessins d'Ernest Cormier pour l'Université de Montréal nous permet également de mieux comprendre l'attachement de l'architecte aux méthodes de travail apprises dans sa jeunesse. Méthodes d'origine française, déjà explicitées dans les directives que l'Ecole du Bâtiment fit parvenir au jeune ingénieur montréalais désireux de réussi. les épreuves d'admission à l'Ecole des Beaux-Arts.

Du travail d'Ernest Cormier à l'Université de Montréal, les premières esquisses qui nous soient parvenues (Figs 197 et 198) sont bien, comme le stipule l'Ecole du Bâtiment, les " première(s) manifestation(s) de la pensée, sous la forme d'un croquis d'ensemble et non de détail " ${ }^{5}$. Ces notations rapides, sans reprise, à l'encre ou au crayon gras, permettent de tester des formules de composition fortement axialisée apprises à l'Ecole des Beaux-Arts. Elles comportent donc une certaine part d'automatisme, mais n'ont toutefois rien d'impressionniste. Chacune indique une implantation précise par rapport au site. Sans avoir à se référer à une quelconque légende, on réalise comment sont distribués et articulés les éléments majeurs du programme-auditorium, laboratoires...

5 CCA ADC- $19 / 3$.
Il ne fait pas de doute que pour Ernest Cormier le travail systématique en plan est à la base de la conception. Lorsqu'on lui demande d'adjoindre un groupe faculté de médecine/hôpital universitaire au plan de septembre 1926, il soumet à ses clients huit configurations différentes pour les ailes de l'hôpital et plusieurs emplacements pour la bibliothèque et l'administration, tout en conservant à l'ensemble sa symétrie et aux axes principaux et secondaires leur cohérence (Figs 188 et 189). Les premières idées de façade consistent en de brèves notations pour mémoire, comme la petite étude sur la version viI (Fig. 188), disposée transversalement selon une pratique courante pour les " esquisses-esquisses "à l'Ecole des BeauxArts. Ensuite, Ernest Cormier produit au crayon des esquisses ombrées très minutieuses (Fig. 199), extrêmement parlantes pour le client ${ }^{6}$. Les études de détail au trait (Fig. 192) sont plus sobres et traduisent de la manière la plus immédiate la recherche d'un "effet plastique intéressant", reposant sur " l'ampleur et l'harmonie des différents volumes, l'équilibre des masses [...] le rythme des proportions" (9).

La prédilection de l'architecte pour des modes de représentation mettant l'accent sur la logique constructive le pousse à produire pour le corps central la grande "coupe-façade " (Fig. 193) dont nous àons déjà noté le rôle de substitut aux traditionnels dessins de présentation. Rien de tel pour démontrer que "les façades sont la conséquence rigoureuse du plan et l'expression franche et loyale des nécessités du programme ", que décor et structure sont en complète harmonie, et pour prouver le bien-fondé de la superposition du vestibule d'honneur et de la bibliothèque. A notre connaissance, ce type de dessin composite, très satisfaisant intellectuellement pour un ingénieur, est généralement peu prisé des architectes anciens élèves de l'Ecole des Beaux-Arts. On trouve un prolongement de cette préoccupation dans la juxtaposition, abstraite mais parfaitement logique, d'une " $1 / 2$ coupe " et d'une " $1 / 2$ élévation ", puis d'un " $1 / 2$ plan toiture " et d'un " $1 / 2$ plan plafond " pour le dessin d'éxécution de la marquise (Fig. 196).

A travers ces quelques remarques, le lecteur aura pris conscience de l'exceptionnelle symbiose entre graphisme et praxis opérée par Ernest Cormier, symbiose que démontrera de manière éclatante la rétrospective du printemps 1990.

6 Une séric importante de ce type d'études de façade subsiste pour le projet d'Annexe au Palais de Justice de Montréal (vers 1920). Voir par exemple cCA 619-x et CCA 269-xx.

1145 Briarcliff Place N.E. Atlanta, Georgia 30306 


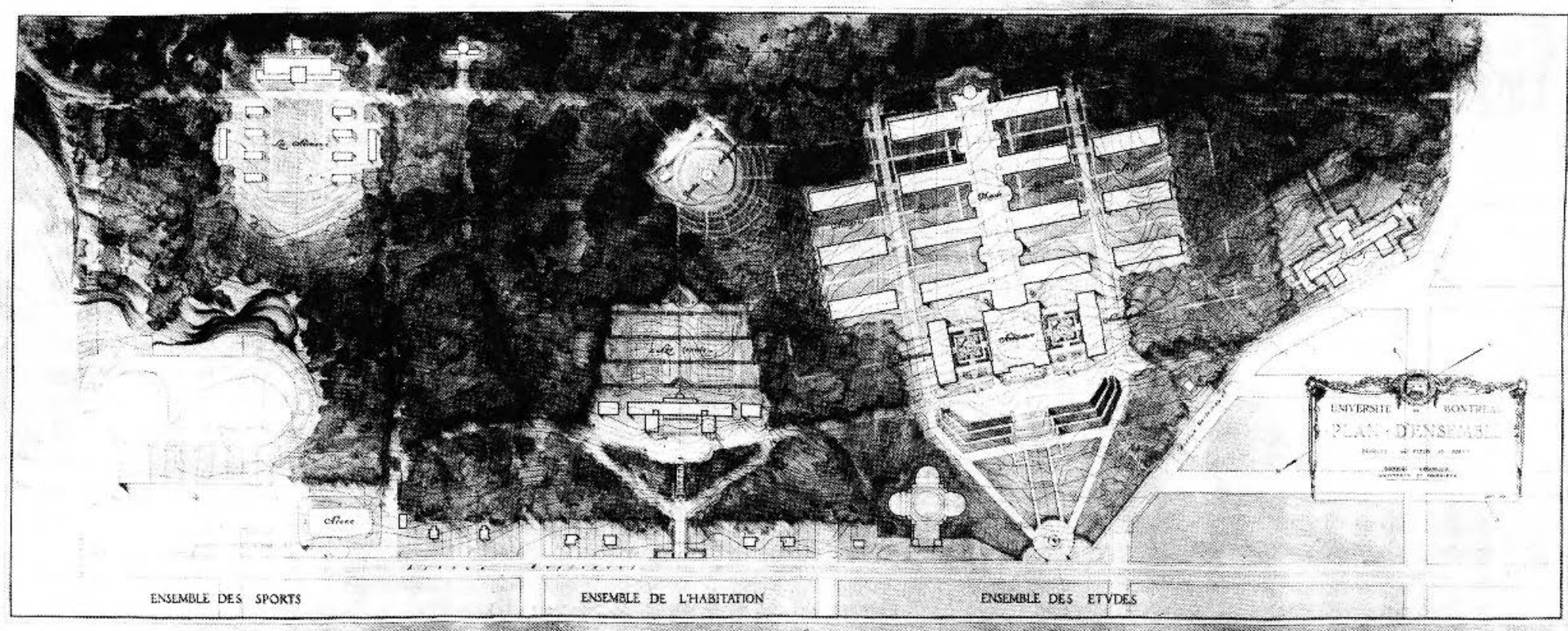

Figure 185. Ernest Cormier, Canada, Montréal 1885-Montréal 1980, Université de Montréal, plan d'ensemble, photostat d'un dessin daté : "septembre 1926 ", tirage ancien, échelle : " 40 pieds au pouce », 46,1 × 104,9 cm. 01 Arc $138 \mathrm{~N}$, Collection Centre Canadien d'Architecture/Canadian Centre for Architecture, Montréal. 


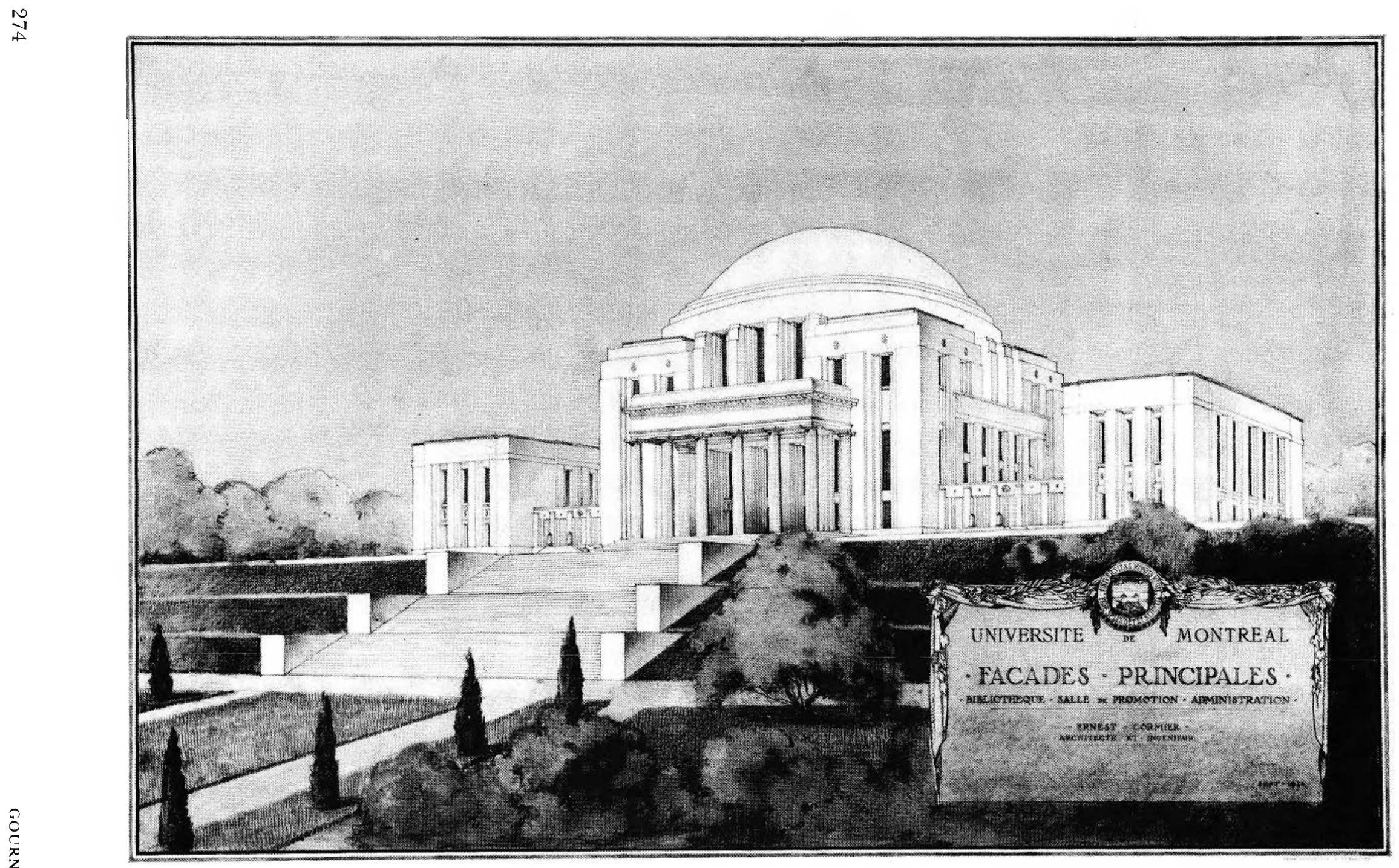

Figuke 186. Ernest Cormier, Canada, Montréal 1885-Montréal 1980, Université de Montréal, façades principales, bibliothèque, salles de promotion, administration, crayon et aquarelle sur carton fort, daté: "septembre 1926 " $67 \times 99,5 \mathrm{~cm} .01$ Arc $137 \mathrm{~N}$, Collection Centrc Canadicn d'Architceture/Canadian Centre for Architecture, Montréal 


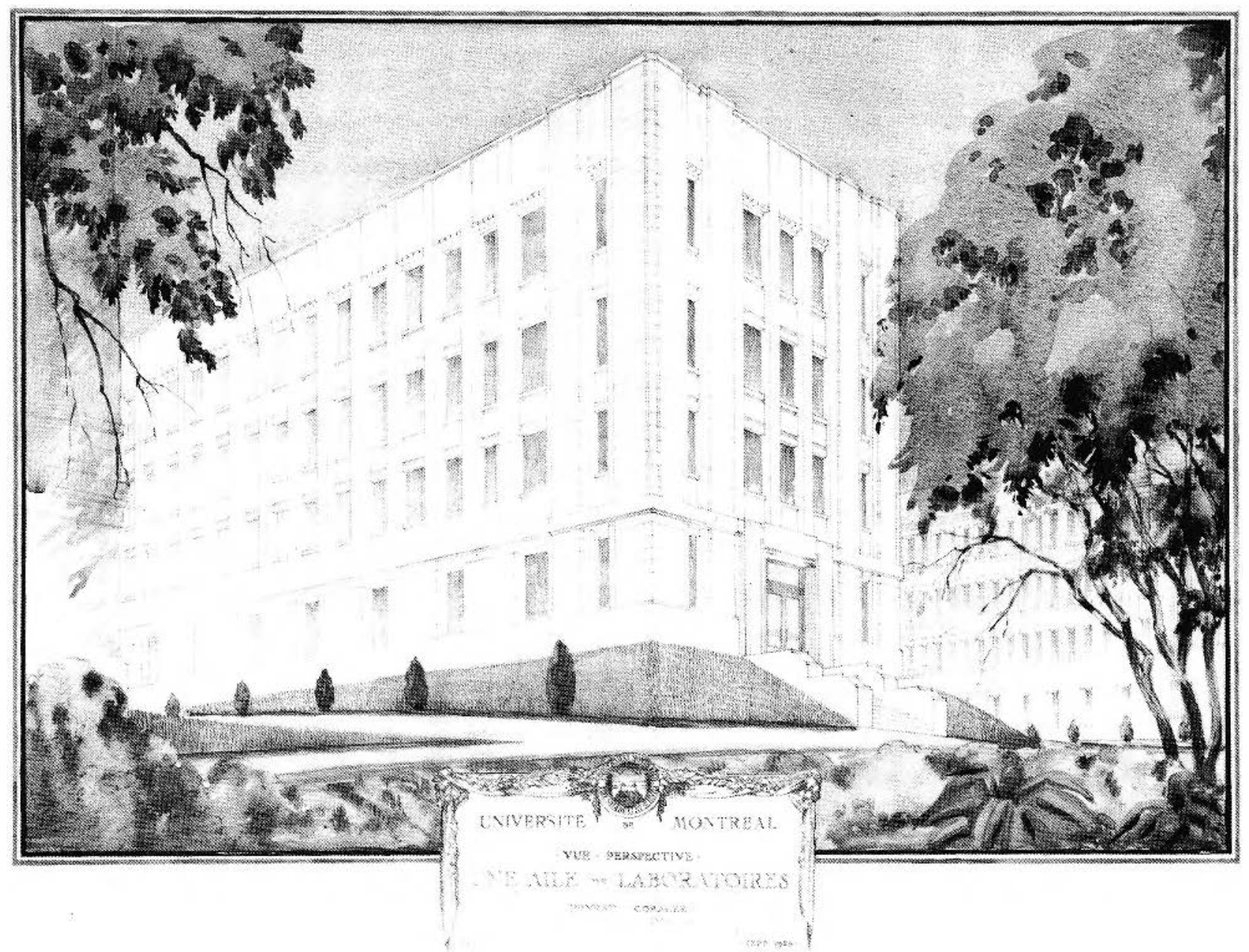

Figure 187. Ernest Cormier, Canada, Montréal 1885-Montréal 1980, Université de Montréal, vue perspective, une aile de laboratoires, crayon et aquarelle sur carton fort, daté : "septembre $1926 », 67 \times 100 \mathrm{~cm}$. 0I Arc 54.N, Collection Centre Canadien d'Architecture/Canadian Centre for Architecture, Montréal. 
Figure 188. Ernest Cormier, Canada, Montréal 1885-Montréal 1980, Université de Montréal, Esquisse numéro VII, mine de plomb sur calque d'étudc, vers janvier/lévrier 1927, $29 \times 40 \mathrm{~cm}$. 01 Arc $16 \mathrm{~N}$, Collection Centre Canadien d'Architecture/Canadian Centre for Architecture, Montréal. 


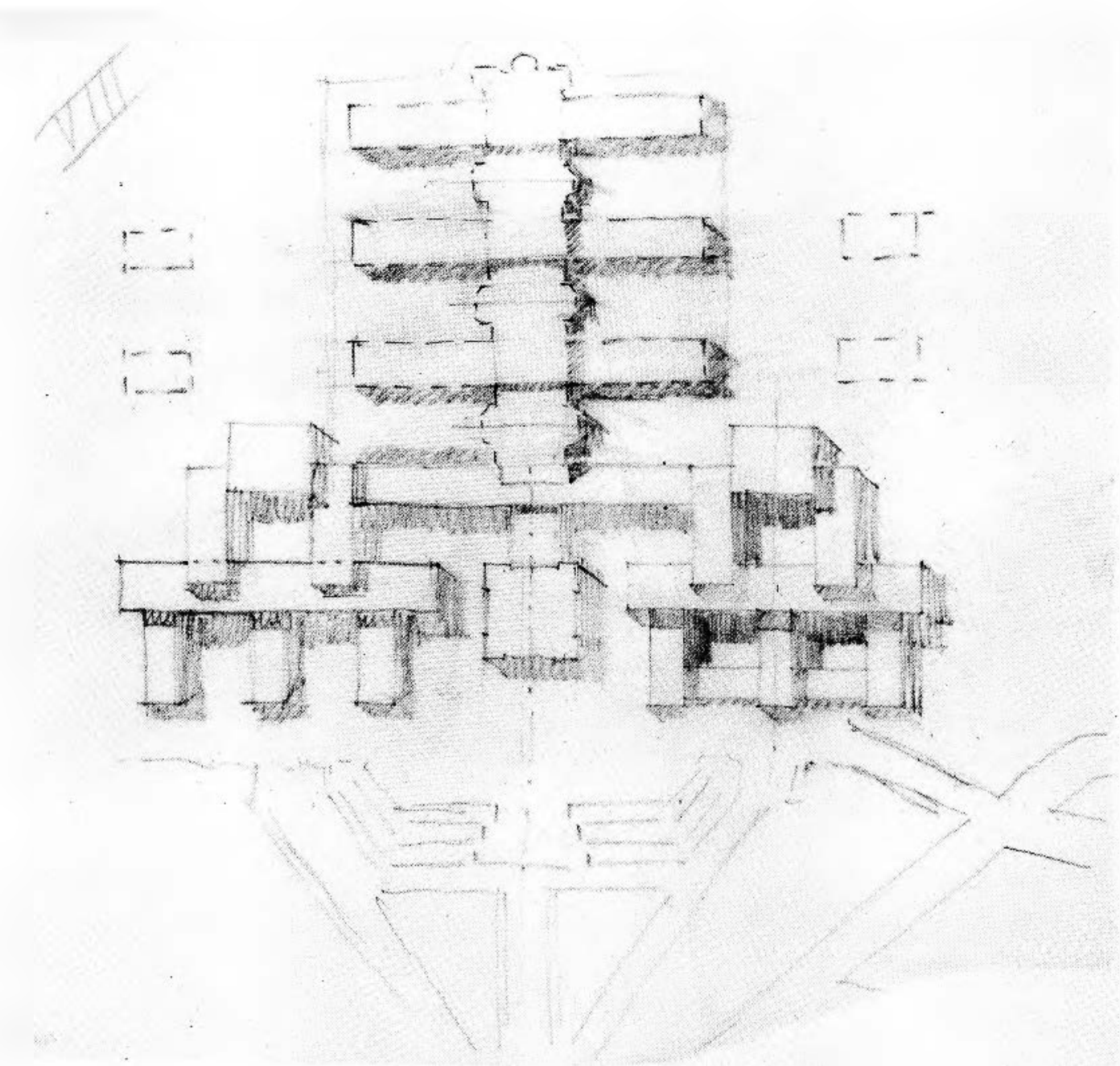

Figlre 189. Ernest Cormier, Canada, Montréal 1885-Montréal 1980, Université de Montréal, Esquisse numéro VIII correspondant au plan adopté le 16 février 1927, mine de plomb sur calque d'étude, $30 \times 32 \mathrm{~cm}$. 01 Arc $17 \mathrm{~N}$, Collection Centre Canadien d'Architecture/Canadian Centre for Architecture, Montréal. 


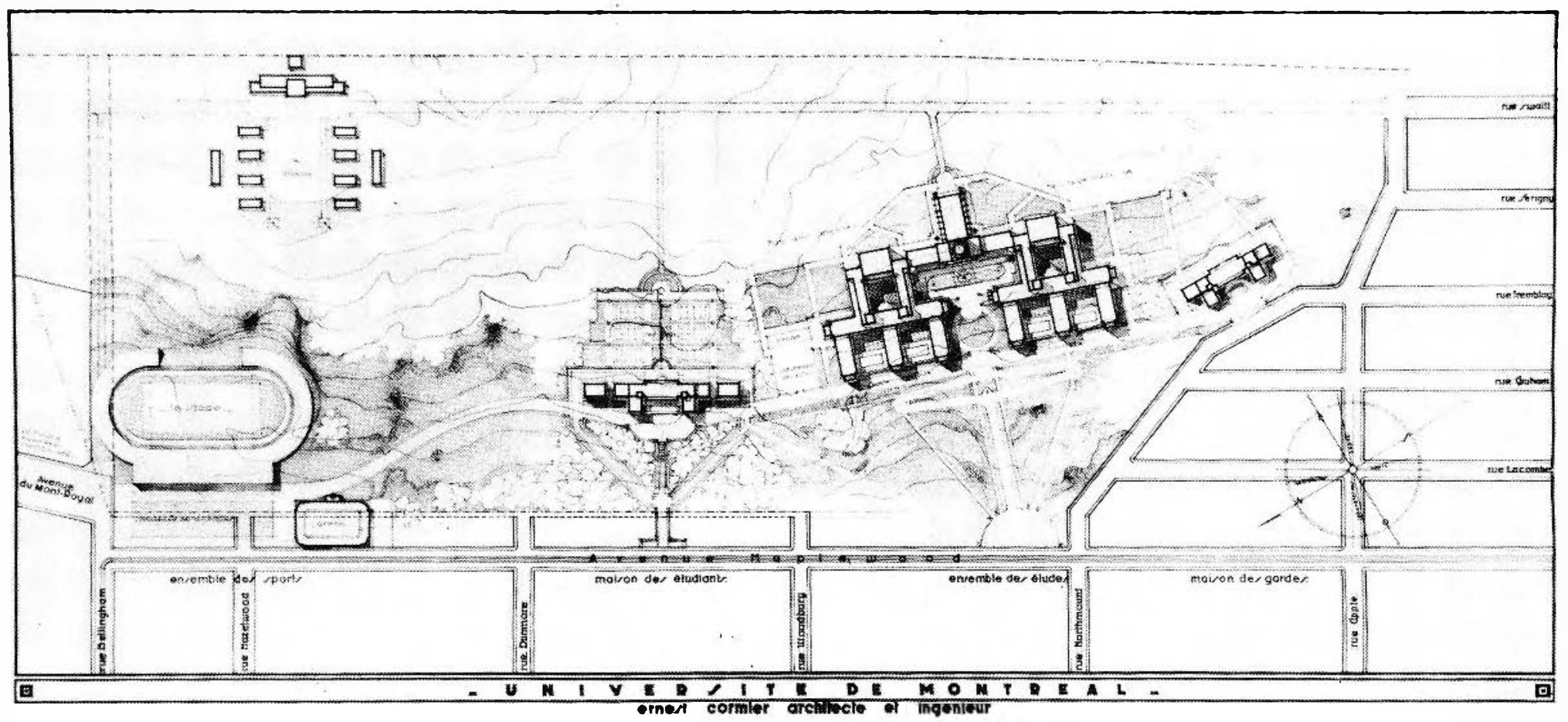

Figure 190. Ernest Cormier, Canada, Montréal 1885-Montréal 1980. Université de Montréal, plan d'ensemble, photostat d'un dessin, tirage anciell, vers 1927,47,5×97,6 cm. () I Arc 139N. Collection Centre Canadicn d'Architecture Canadian Centre for Architecture, Montréal. 


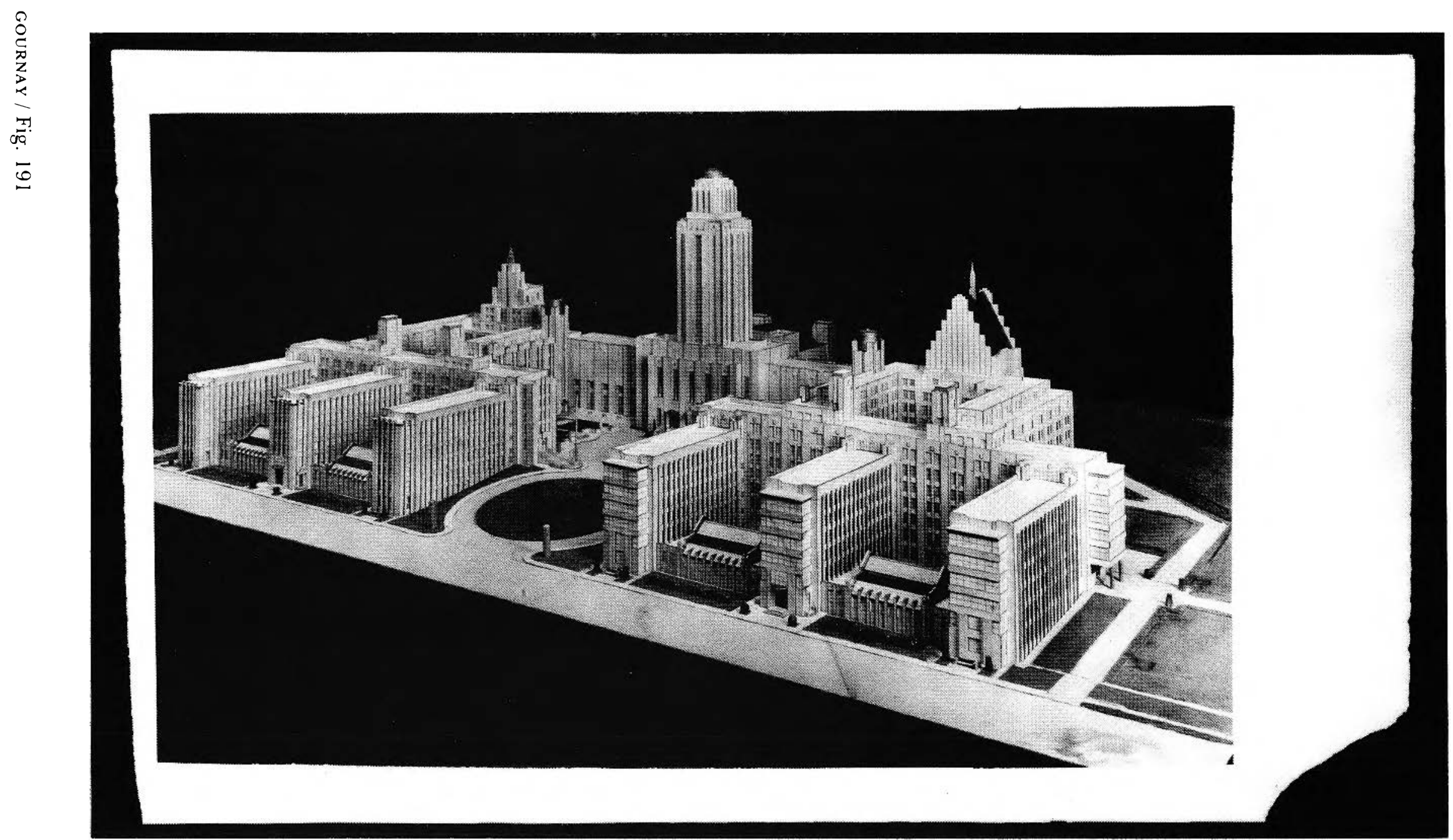

Figure 191. Samuel Jack Hayward, Angleterre(?)-Floride 1956, actif à Montréal 1917-1945, Photographie de la maquette d'étude d'Ernest Cormier. Université de Montréal, façade principale, parti définitif, epreuve argentique à la gelatine, vers 1930, 52,5 × $88 \mathrm{~cm} .01$ Arc 52N, Collection Centre Canadien d'Architecture/Canadian Centre for Architecture, 


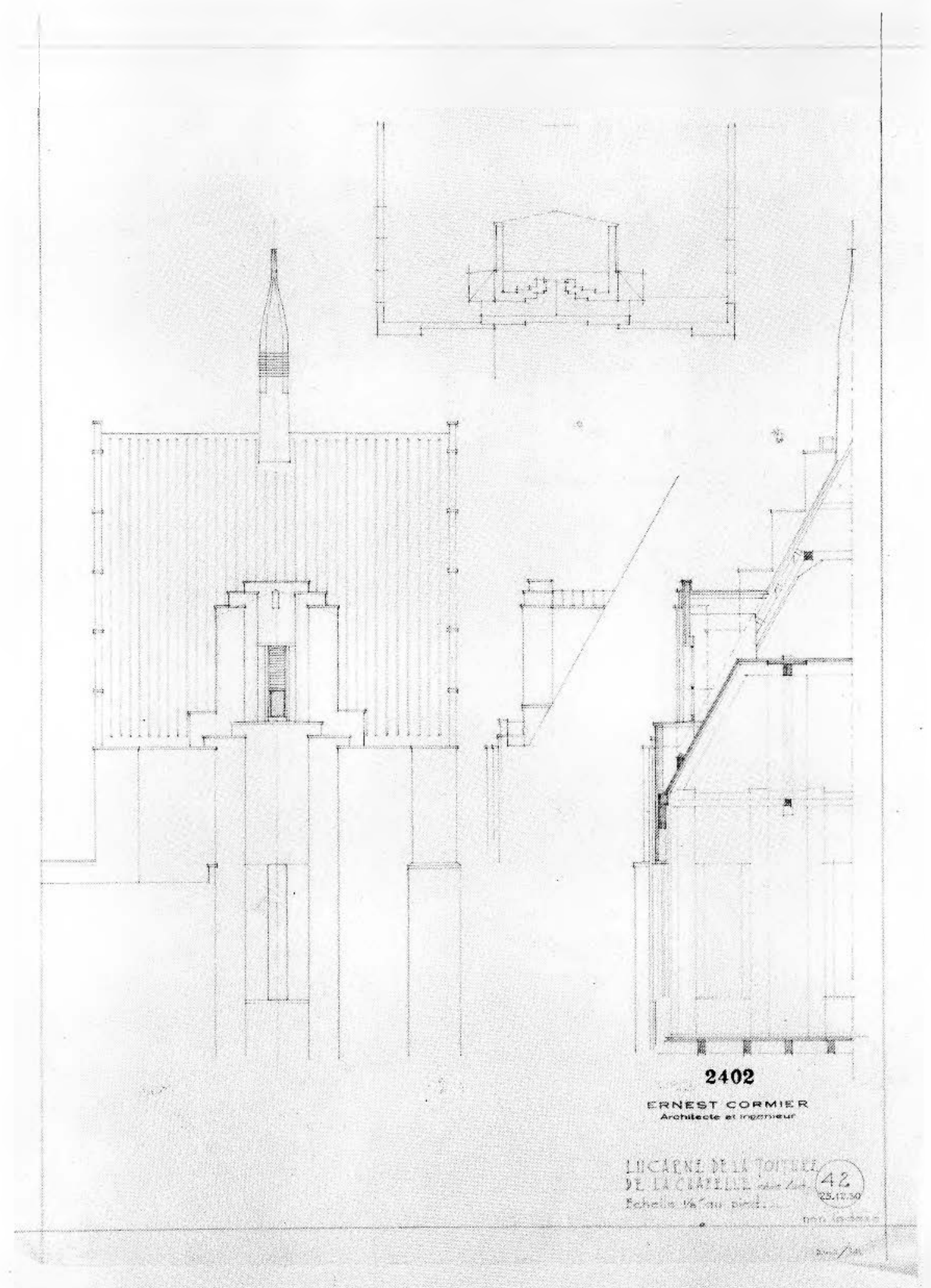

Figure 192. Ernest Cormier, Canada, Montréal 1885-Montréal 1980, Université de Montréal, lucarne de la toiture de la chapelle, côté sud, dessin accompagné des initiales E.C. dans le registre consignant les dessins d'exécution, plan, coupe, élévation, mine de plomb sur calque, daté : " 25 septembre 1930 ", échelle : "18" au pied ", $61 \times 44 \mathrm{~cm} .01 \mathrm{Arc} 216 \mathrm{~N}$, Collection Centre Canadien d'Architecture/Canadian Centre for Architecture, Montréal. 


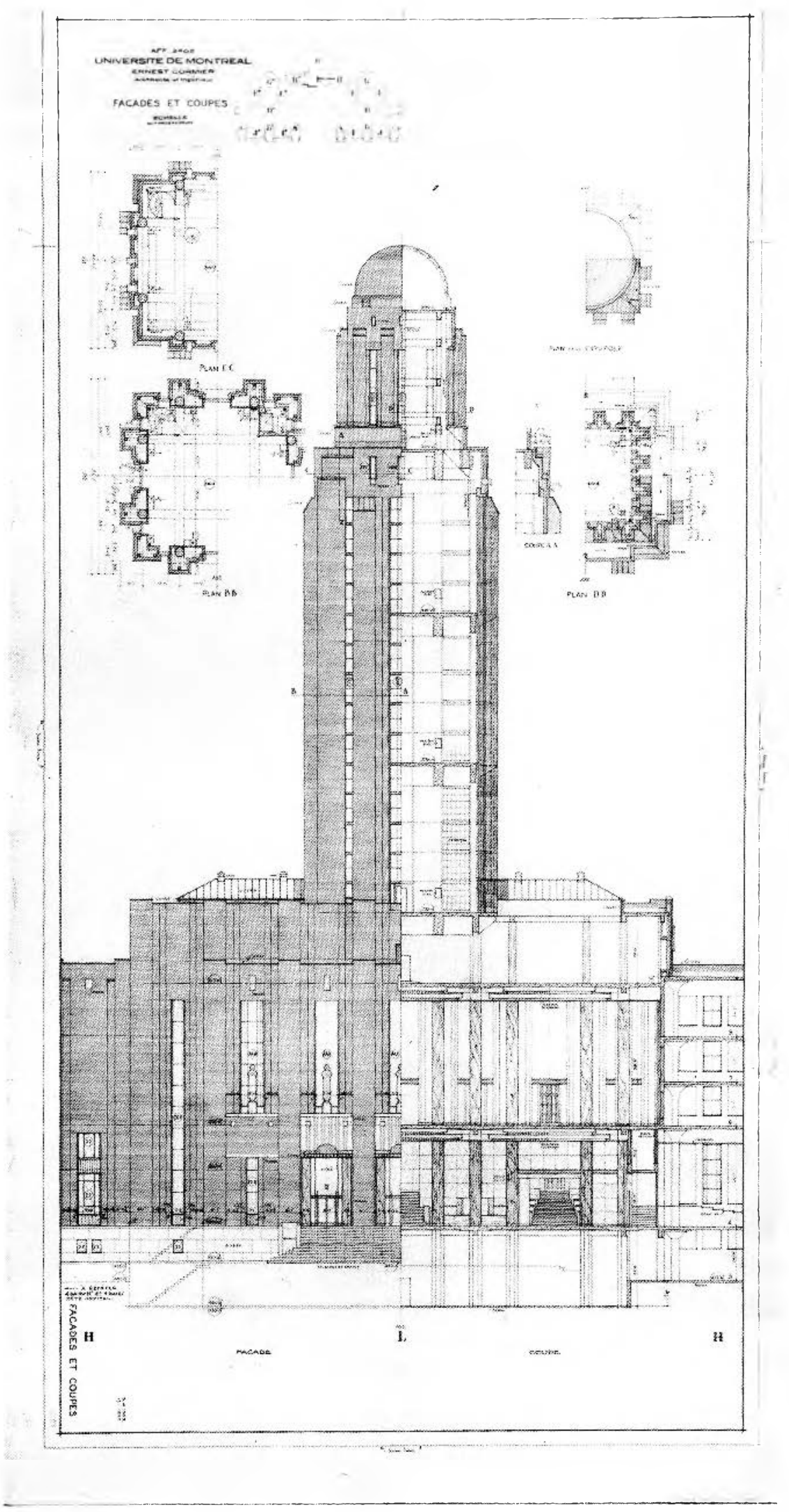

Figure 193. Ernest Cormier, Canada, Montréal 1885-Montréal 1980, Université de Montréal, coupe-façade sur le vestibule d'honneur et la tour, encre sur toile, daté : 5 avril 1929 et 17 janvier 1930 ", 65,5 $\times 130 \mathrm{~cm}$. 01 Arc $140 \mathrm{~N}$, Collection Centre Canadien d'Architecture/Canadian Centre for Architecture, Montréal. 


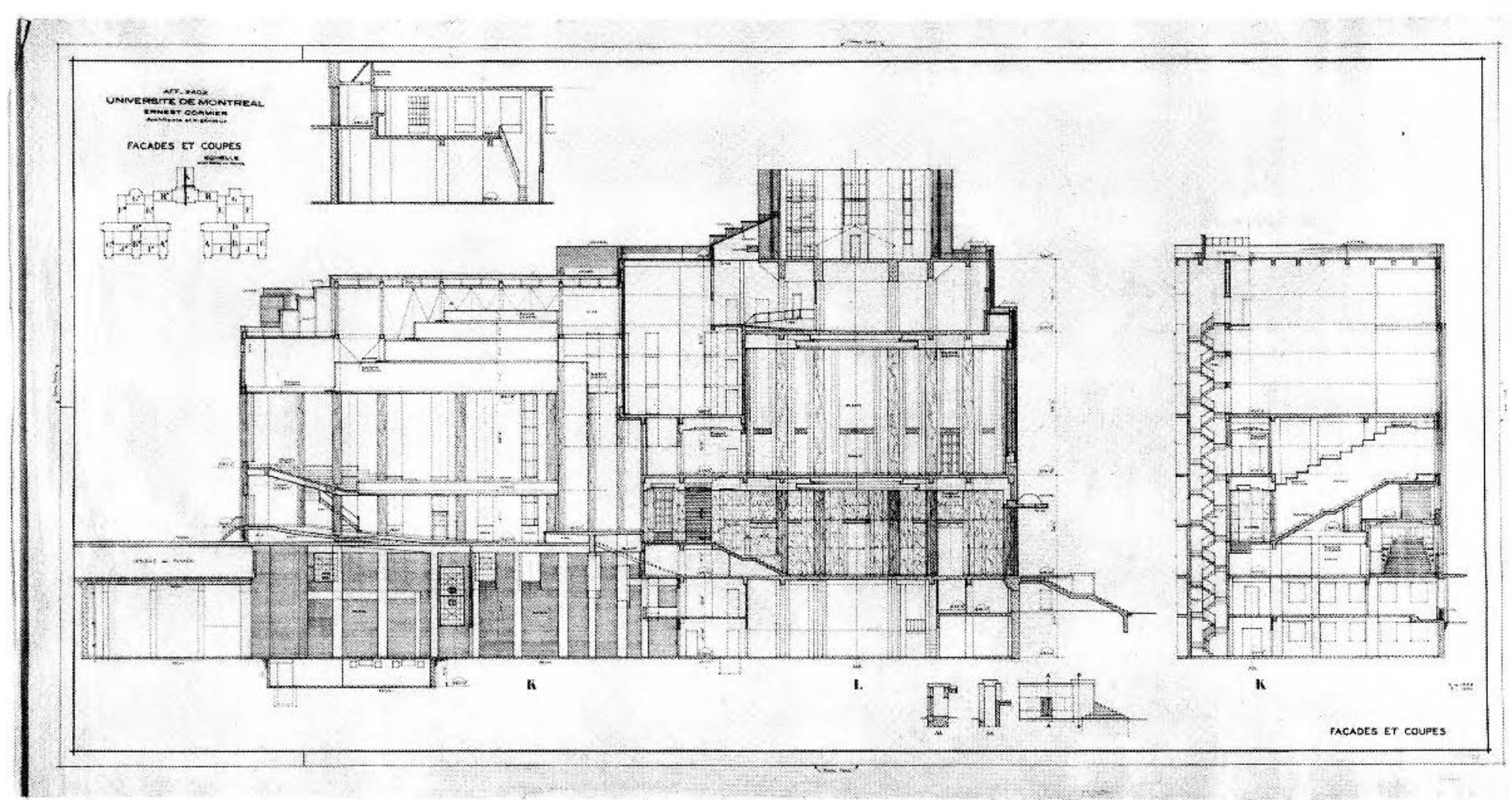

Figure 194. Ernest Cormier, Canada, Montréal 1885-Montréal 1980, Université de Montréal, coupe-façade sur le vestibule d'honneur et l'auditorium cncre sur toile, daté : 5 avril 1929 et 17 janvier $1930 » 65,6 \times 138,3 \mathrm{~cm}$. 01 Arc I4I N, Collection Centre Canadien d'Architecture/Canadian Centre for Architecture, Montréal. 


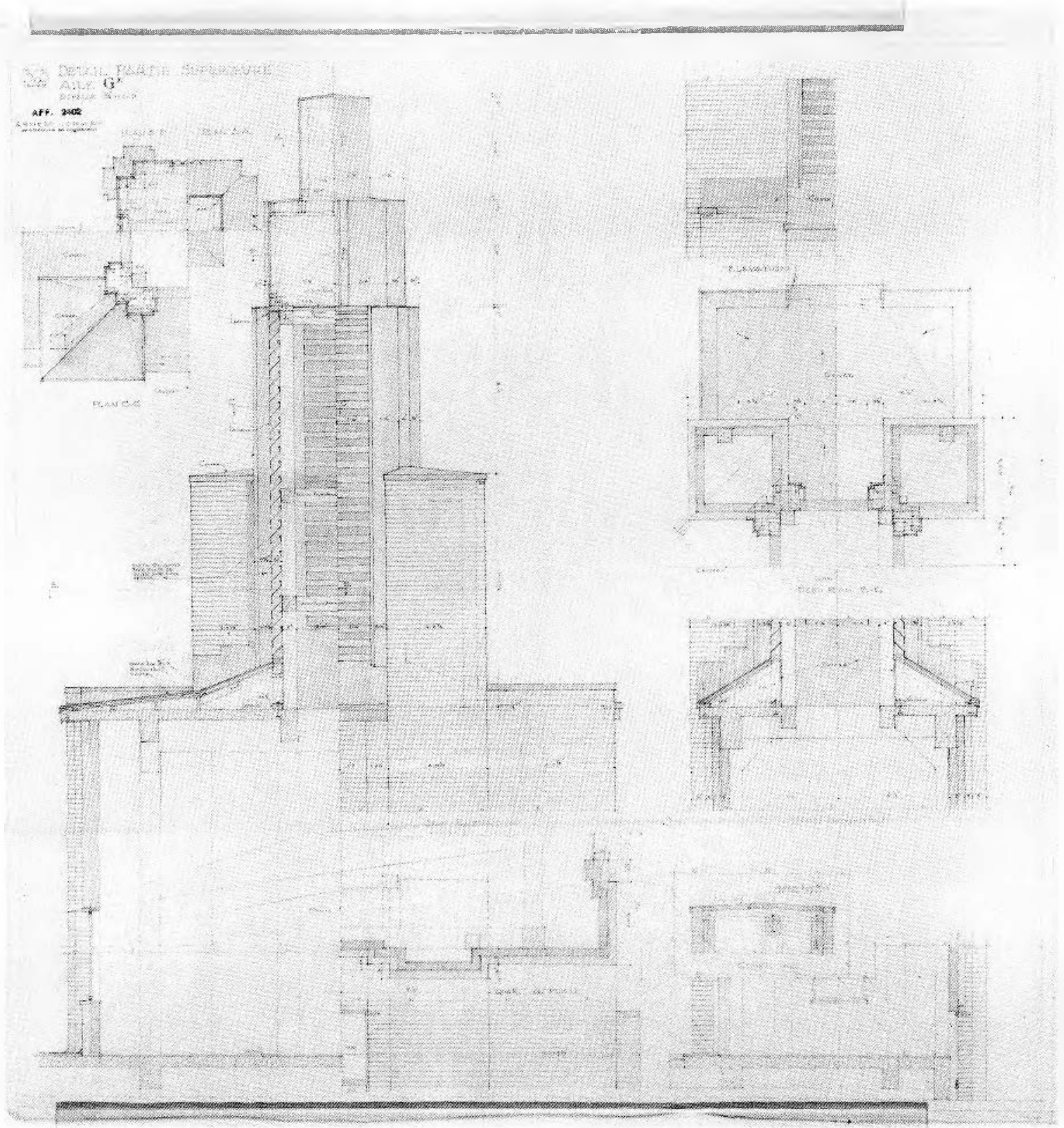

Figure 195. Willford Arthur Gagnon, Canada, Montreal, 1878-?, dessinateur, et Ernest Cormier, Canada, Montréal 1885-Montréal 1980, architecte. Université de Montréal, détail de la partie supérieure de l'aile $G$, dessin accompagné des initiales W.A.G. dans le registre consignant les dessins d'exécution, plan, coupe, élévation, mine de plomb sur calque, daté : " 30 octobre 1931 ", échelle : "34" =1'0" ", 100×94 cm. 01 Arc 218N, Collection Centre Canadien d'Architecture/Canadian Centre for Architecture, Montréal. 


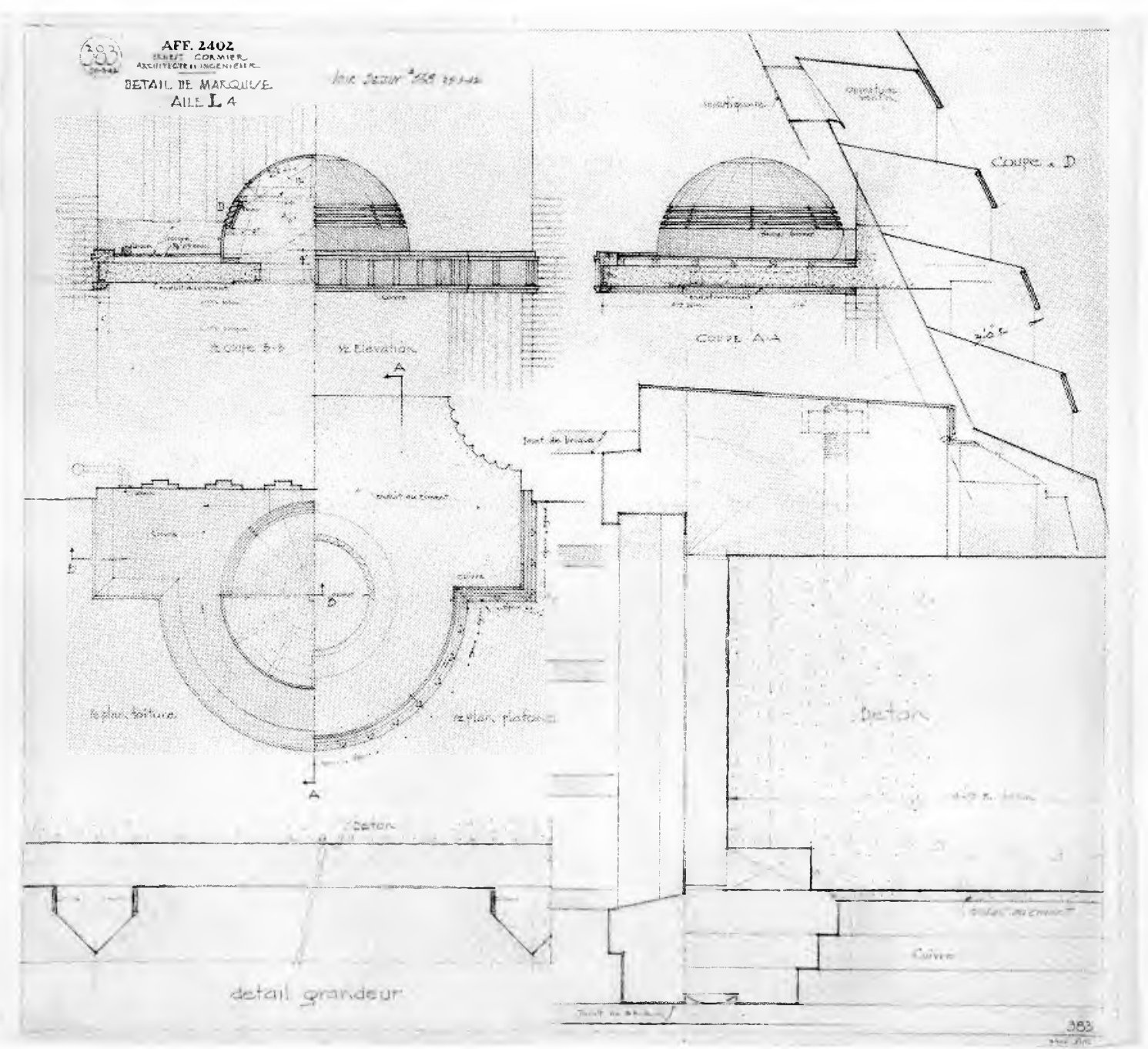

Figure 196. Willford Arthur Gagnon, Canada, Montreal, 1878-?, dessinateur, et Ernest Cormier, Canada, Montréal 1885-Montréal 1980, architecte, Université de Montréal, détail de la marquise de l'aile L4, dessin accompagné des initiales W.A.G. dans le registre consignant les dessins d'exécution, plan, coupe, élévation et détail, mine de plomb sur calque, daté : " 28 mars 1942 ", échelle : " $34^{\prime \prime}=1{ }^{\prime} 0$ " ", $100 \times 94 \mathrm{~cm}$. 01 Arc 178N, Collection Centre Canadien d'Architecture/Canadian Centre for Architecture, Montréal. 

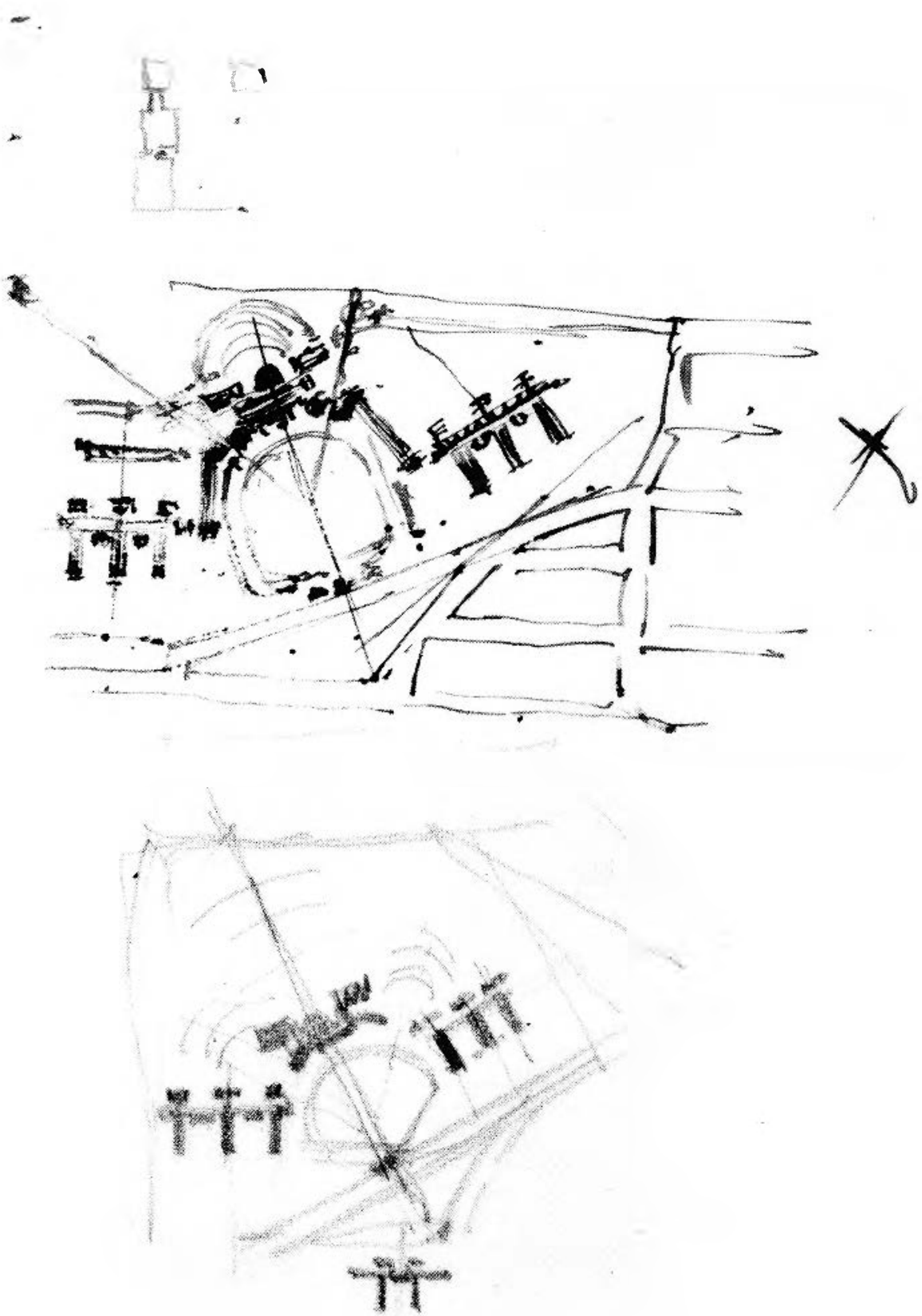

Figure 197. Ernest Cormier, Canada, Montréal 1885-Montréal 1980, Deux esquisses pour le projet de l'Université de Montréal, encre sur calque, vers avril 1924, 17 × $13 \mathrm{~cm} .01$ Arc $31 \mathrm{~N}$, Collection Centre Canadien d'Architecture/ Canadian Centre for Architecture, Montréal. 


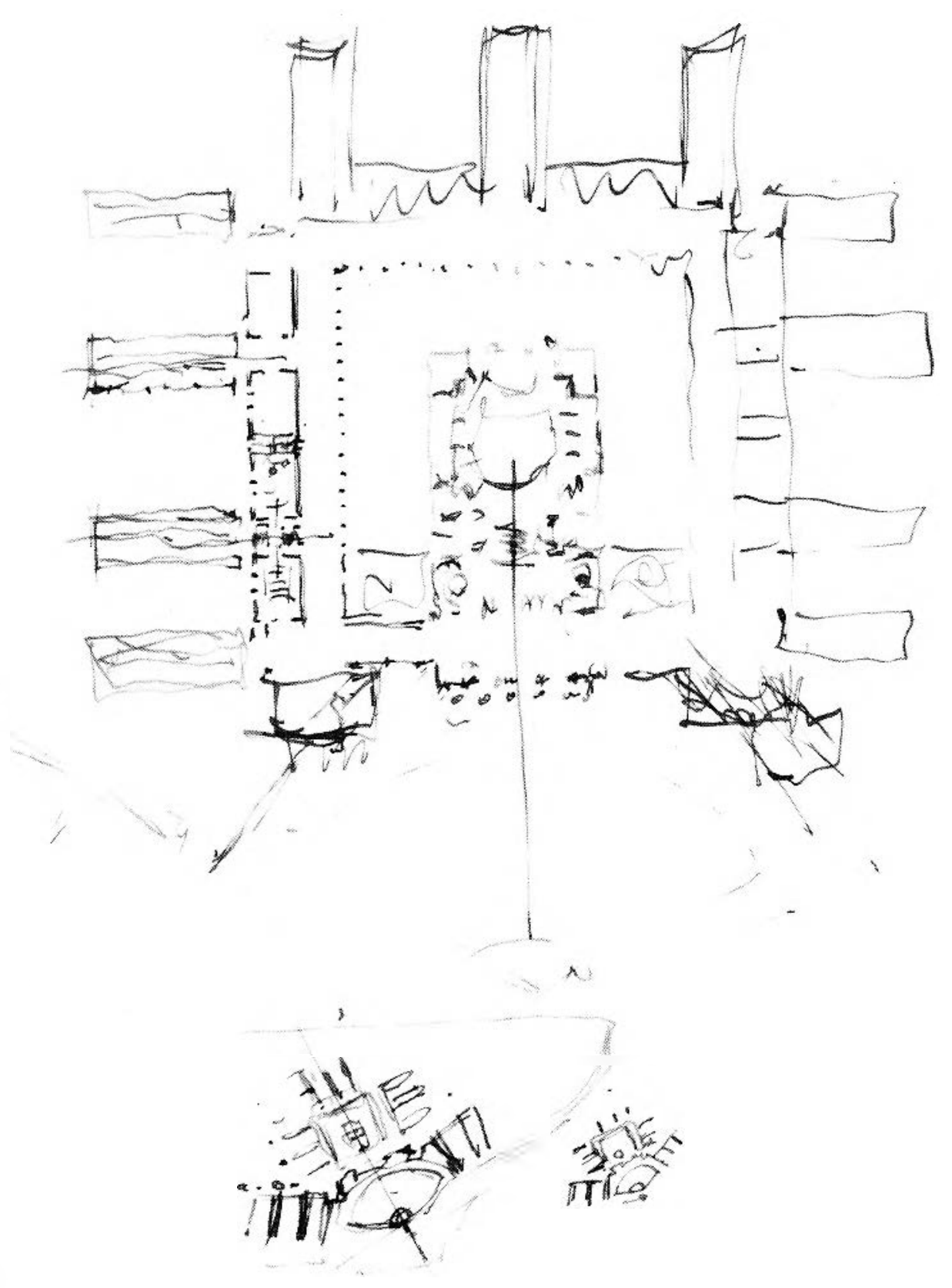

Figure 198. Ernest Cormier, Canada, Montréal 1885-Montréal 1980, Deux esquisses pour le projet de l'Université de Montréal, encre sur calque, vers avril 1924, $28 \times 21 \mathrm{~cm}$. 01 Arc $32 \mathrm{~N}$, Collection Centre Canadien d'Architecture/ Canadian Centre for Architecture, Montréal. 

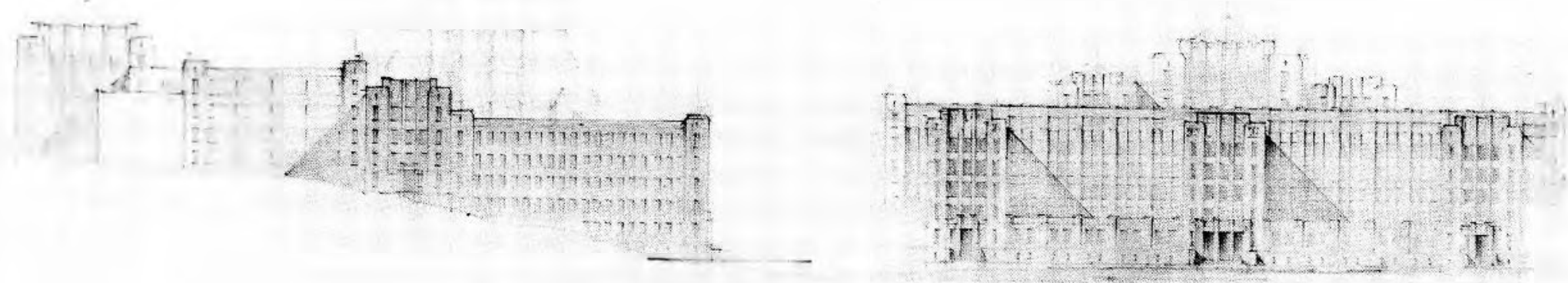

Figure 199. Ernest Cormier, Canada, Montréal 1885-Montréal 1980, Université de Montréal, étude, deux coupes-façades à petite échelle, mine de plomb sur calque d'étude, vers février 1927, 33,5 ×75 cm. 01 Arc 58N, Collection Centre Canadien d'Architecturc/Canadian Centre for Architecture, Montréal. 